\title{
Does the study of feeding behaviour benefit from a teleonomic framework?
}

\author{
Ilias Kyriazakis ${ }^{1}$ and Jon E. L. Day ${ }^{2}$ \\ ${ }^{1}$ Animal Biology Division, Scottish Agricultural College, West Mains Road, Edinburgh EH9 3JG, UK \\ ${ }^{2}$ ADAS Terrington, Terrington St. Clements, King's Lynn, Norfolk PE34 4PW, UK
}

\begin{abstract}
In this paper we respond to the criticisms of Provenza et al. (1998) that our framework of learning and feeding motivation (Day et al. 1998) resorts to higherorder goals, which cannot be falsified by experimentation. We assert that in order to be able to predict the feeding behaviour of animals we first need to understand what they are trying to achieve (i.e. invoke teleonomy). We then detail our framework in such terms that one could envisage experiments that could quantitatively test its predictions. We contend that the framework of 'the self-organization of behaviour' proposed by Provenza et al. (1998) cannot lead to such quantitative predictions, since it is invoked to describe feeding behaviour of animals $a$ posteriori. It is our own desire, by contrast, to assess feeding behaviour a priori, which leads us to propose and defend our framework of learning and feeding motivation.
\end{abstract}

\section{Introduction}

We have recently proposed (Day et al. 1998) a framework to account for the feeding behaviour of animals in terms of learning and motivation. Our framework was based on two propositions: (i) feeding behaviour is directed towards achieving a goal, and (ii) animals are motivated to actively sample food items in order to assess whether they are nutritionally beneficial or harmful. Our framework has been criticized by Provenza et al. (1998) in this issue, on the grounds that we resort to hypothetical agencies, such as motivation, and invoke higher level goals to explain feeding behaviour. For Provenza et al. (1998) feeding behaviour is "a selforganizing system, which evolves from non-linear relations among the components of the system and the rules for their interactions"; "it arises from simple rules which operate at levels of resolution from cells and organs to individuals...". Their view of the self-organization of behaviour leads to the implicit suggestion that feeding behaviour is essentially unpredictable, since it can only "be explained in terms of actual rather than expected behaviour": "explanation is reduced to description and the notion of function substituted for that of causation" (Mach, 1960).

We are receptive to the above criticisms, especially to the one that recourse to inferred higher-level goals cannot be falsified by experimentation, having ourselves applied the same criticisms to others in the past (Emmans \& Kyriazakis, 1995). However, we are mainly 
interested in understanding and predicting the feeding behaviour of farm animals, rather than simply describing observations; after all, one can claim to understand how a system operates only if one can successfully predict its behaviour. We appreciate that our approach contrasts with the post hoc, descriptive approach of Provenza and his coworkers. For example, in a recent paper of Villalba \& Provenza (1997), the preferences and aversions of lambs for flavoured foods associated with different doses of nitrogen were investigated. It is stated there that "we did not know the concentrations of $\mathrm{N}$ required to modify preference. Thus for all our experiments the level of $\mathrm{N}$ administered varied (and was confounded) with period of conditioning". This statement seems to ignore the plethora of information on the postingestive consequences created by $\mathrm{N}$ administration in sheep (e.g. as summarized by Ørskov, 1988).

Here, we will contest that in order to be able to predict feeding behaviour, it is both useful and necessary to resort to a teleonomic framework. Therefore, we will first summarize our framework of learning and motivation in terms that have not been previously made explicit in Day et al. (1998), because it was not considered necessary to do so. We will then highlight the shortcomings of the framework to predict feeding behaviour under certain circumstances. Lastly, we will discuss why the concept of exploration needs to be invoked to complement the framework, in order to account for such contradictions in the outcome of feeding behaviour.

\section{A teleonomic framework applied to feeding behaviour}

A behaviour or a process is referred to as teleonomic when it is characterized by a goal directedness which is controlled by a programme (Mayr, 1964). It thus depends on the existence of some end point, goal or terminus which is foreseen in the programme that regulates the behaviour. The programmes which control teleonomic behaviours in organisms are either laid down in their genetic make-up or are constituted in such a way that they can incorporate additional information acquired through learning, conditioning, or other experience; the evolution of programmes could thus be seen as favoured by natural selection. Nowadays the use of teleonomic language in biology is considered legitimate, since it neither implies a rejection of a physicochemical explanation nor does it imply noncausal explanation (Hull, 1974; Mayr, 1988).

However, caution should be exercized in the invocation of teleonomic language and especially in the use of the term goals. End points or goals of behaviour should be considered specific rather than generalized processes. Specific goals can lead to explicit theories which can be tested, criticized and eventually replaced. It is obvious that the same cannot apply to generalized goals of behaviours, and in that respect we share the reservations of Provenza et al. (1998) that recourse to higher order goals cannot be falsified by experimentation and, therefore, they are empty concepts.

In our paper (Day et al. 1998) we briefly described the teleonomic framework within which feeding behaviour was placed and its goal directedness was asserted. It was stated there that "it is beyond the scope of this review to discuss the nature of this goal, or goals ...", but this seems to have made it open to criticism (Provenza et al. 1998). For this reason the nature of the goals of the feeding behaviour of animals will be made explicit here; they have been used as such in the work of the first author of this paper to make specific predictions of what animals will do when they are offered ad lib. access to a food of a given quality, or to two or more foods as a choice (e.g. Kyriazakis et al. 1991; Kyriazakis \& Oldham, 1993; Emmans \& Kyriazakis, 1995; Kyriazakis, 1997).

Animals have been assumed to have output goals, such as maximum rates of growth, or rates of egg or milk production, which they are seeking to achieve. In order to be successful 
they need resources from their environment, including those such as energy and amino acids that they can get only from their food, at the rates needed to support the level of performance that they seek and no more. Consequently, when animals are given access to two or more foods as a choice, they will select from these a diet which allows them to perform as well as they could on any mixture of these foods and, at the same time, will minimize excess of nutrient intake. The above assumptions are expected to lead to specific expectations in the feeding behaviour of animals only if the animal has some prior knowledge of the postingestive consequences of the foods available in their environment. What is food and how much of a specific food should be eaten is learned mainly by the young who have an open programme for this type of information. Thus, this particular component of the feeding behaviour was not acquired through natural selection and yet it is considered to be an integral part of teleonomic behaviour.

The above framework can be seen as having a heuristic value in pointing towards the nature of the goals and the problems that can arise when constructing a teleonomic framework of feeding behaviour in animals. In order, for example, to predict the feeding behaviour of animals on a high quality, nonconstraining food, a description of the maximum output targets they are seeking to achieve is necessary. Solutions to this problem are currently available (e.g. Kyriazakis \& Emmans, 1998). The framework is put in such terms that one could envisage experiments that could test quantitatively its predictions. The criticism that we have constructed a theory which cannot be falsified by experimentation is therefore unwarranted.

\section{Apparent deficiencies of the framework}

Our framework makes specific predictions of the feeding behaviour of animals when they are given access to one or more foods, the nutritional properties of which and hence postingestive consequences are known to them. The first problem then is how the framework can be enriched to account for the response of naive animals towards novel food resources; the term novel is used here broadly, to account for both truly novel foods and for foods the properties of which change over time. For this purpose we have proposed that animals are actively motivated to explore novel stimuli, including food items, and called this motivation intrinsic exploration. For an animal without any prior feeding experience all environmental stimuli could be seen as potential food resources. This view does not seem to be at odds with that of Provenza et al. (1998) on how animals identify new foods, mainly by observing their mothers or experienced peers. We consider that the latter merely directs intrinsic exploration towards certain food items. However, consuming only what the mother does would constitute, in some instances, a failure to achieve the young's output targets (Dardaillon, 1989).

A consequence of the framework is that the animals will be expected to learn to optimize their feeding behaviour in a range of feeding situations. When, for example, they are offered a choice between two or more foods neither of which, nor their combination, allows them to reach their output targets, they would be expected to consume a diet which would consist solely of the least constraining food. As we have stated (Day et al. 1998), this does not seem to be in agreement with the outcome of experiments where such a choice is offered (a number of examples has been given in our previous paper). The second problem then is to indicate how our framework could be used to make its predictions consistent with such outcomes. We have proposed that animals are always motivated to continue sampling all available foods, even when their nutritional properties are known to them, and called this motivation extrinsic exploration. We have offered reasons why such a behaviour would be consistent with a teleonomic framework, mainly because the nutritional properties of food items are expected to vary 
both spatially and temporally, even when the other properties (e.g. sensory) of the foods do not change (Bazely, 1990).

The use of intrinsic and extrinsic exploration allows us to account for the feeding behaviour of animals, at least qualitatively, within the framework we have outlined above. The question, however, is whether such constructs could be put in explicit, quantitative terms, similar to those in which the rest of the framework has been constructed. Otherwise, they would remain cognitive constructs without much scientific value, as Provenza et al. (1998) have suggested.

\section{Towards quantification of intrinsic and extrinsic exploration}

We have viewed intrinsic exploration as a separate behaviour which competes with other behaviours, including feeding, for its expression. This implies that intrinsic exploration would decline in animals which have to spend a substantial effort on, for example, sexual behaviour or harvesting a food (Friggens et al. 1998). On the other hand it would be expected to increase when, for example, little time is devoted to feeding owing to imposed food restriction. The latter suggestion is consistent with the observations on food restricted animals (Day $e$ t al. 1986) and ruminants offered a highly digestible, easy to harvest food (Cooper et al. 1994); they spend significantly longer engaged in activities such as chewing or interacting with stimuli of little or no nutritional significance. Attempts to model related behaviours which compete with each other for their expression, such as feeding and drinking, already exist in the literature (e.g. Sibly, 1975; Sibly \& McCleery, 1976); these could easily be extended to predict the interactions between feeding behaviour and intrinsic exploration. We have nothing new to offer in this respect.

The situation of modelling extrinsic exploration and making quantitative predictions that are consistent with the feeding behaviour of animals given access to more than one constraining food as a choice appears to be slightly more complex. The simplest starting assumption to make is that animals in such situations will always explore or sample the least attractive option; the latter is defined as the most constraining food of those on offer. A consumption of $<5 \%$ of the total intake as the most constraining food for an animal at a particular point in time is suggested; it is further proposed that this baseline of extrinsic exploration should be independent of animal state (i.e. feeding motivation). These suggestions are consistent with the diet selection of animals given a choice between two foods which are both above their requirements (i.e. contain nutrients in excess; Kyriazakis et al. 1990; Kyriazakis \& Oldham, 1993). They point towards the ability of animals to discriminate against nutrient excesses, which could be seen to act as toxins (Kyriazakis et al. 1998; Arsenos \& Kyriazakis, 1998).

It is, however, to be expected that the more nutritionally similar the available feeding options become, the less able the animal will be to discriminate between them, and hence the Weber law would apply, i.e. increased consumption of the least attractive feeding option would be observed. This will apply particularly to foods which supply nutrients below the animal's requirements (Kyriazakis et al. 1990; Arsenos \& Kyriazakis, 1998). The above suggestions on the diet selection of animals offered a choice between two foods with a different relative nutrient deficit or excess are summarized graphically on Fig. 1. The figure essentially replicates the recently proposed model of Arsenos \& Kyriazakis (1998). The last point to consider is whether the ability to discriminate between two or more options, neither of which is able to meet the animal's requirements in relation to its output target(s), would be affected by animal state. Recently, Kyriazakis (1997) has suggested that the more feeding motivated or nutrient deprived the animal is, the more able it would be to discriminate between feeding options. This would imply that extrinsic exploration would decline, but not below its baseline level, in 


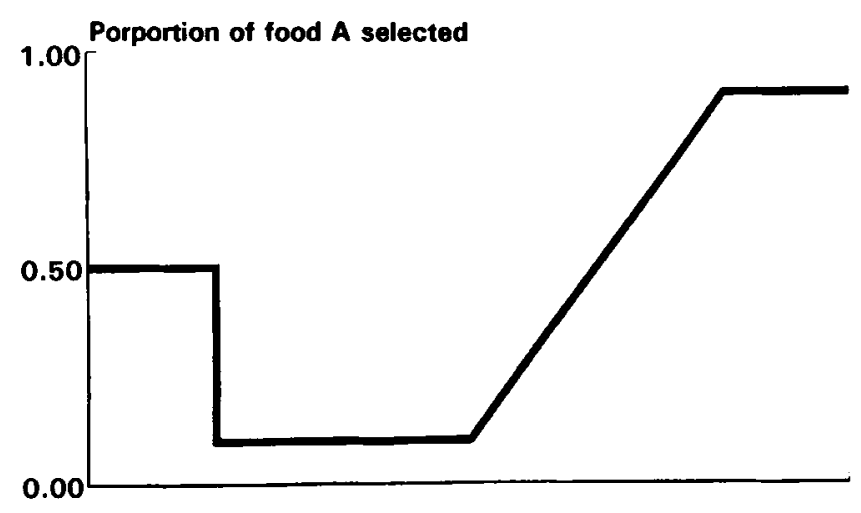

Food B nutrient content

Figure 1. A proposed model for the diet selection of an animal offered a choice between two foods ( $A$ and $B$ ). The nutrient composition of food $A$ is constant and below the animal's requirements, the nutrient composition of food $B$ varies (starting point: similar composition to food A). The diet selection is expressed as the proportion of food A selected ( $g$ food $A / g$ total intake (food $A+$ food $B$ )).

feeding motivated animals offered choices between foods which are unable to meet their requirements. These suggestions are consistent with a recent model which aimed at predicting the feeding behaviour of animals offered the opportunity to work for their food or to obtain identical food freely (Inglis et al. 1997).

In the above we have discussed how intrinsic and extrinsic exploration can be put in such terms that would complement our teleonomic framework and lead to predictions on the foraging behaviour of animals. Our approach is far from "a re-statement of the observation".

\section{Time scales of feeding behaviour}

Here we will only briefly touch on the time scales of the feeding behaviour, as viewed by Provenza et al. (1998) and by ourselves. This is relevant to predictions of feeding behaviour, and therefore of direct relevance to this paper. It is explicitly stated by Provenza et al. (1998) that their framework applies to short term feeding behaviour, i.e. within a feeding bout or a meal. They contend that animals are equipped with mechanisms which enable them to detect changes in their internal state as a consequence of food ingestion, and hence modify their behaviour within a feeding bout. Unfortunately, the evidence they provide to support their view (Provenza, 1995) is precisely that which refutes their arguments: short term, systemic fluctuations that occur in the profiles of metabolites or hormones during a meal appear to relate very little to modifications of feeding behaviour (de Jong, 1981).

There is now sufficient evidence to suggest that animals are able to reach the same outcome in their feeding behaviour (in terms of feed intake and diet selection) through very different strategies. For example, similar pigs achieve the same daily food intake through either large, infrequent meals or small, but frequent ones (Nielsen et al. 1995). Lactating cows achieve the same daily composition in their diet selection, through completely different diurnal patterns of diet selection (Tolkamp \& Kyriazakis, 1997). For further examples of this the reader is referred to Tolkamp et al. (1998). We interpret the above examples as evidence in support of 
the plasticity of short term feeding behaviour, which is a device by which animals exploit effectively their feeding environment (Kyriazakis, 1997). Furthermore, it would be very difficult to construct a system which would predict such very different short term feeding strategies adequately.

In contrast, there is some strong evidence that long term feeding behaviour relates very closely to long term changes in an animal's internal state (as reviewed by Kyriazakis, 1997). Animals seem to respond to long term, significant changes in their internal state by modifying their feeding behaviour. This monitoring and control would allow them to achieve their goals or output targets, which have been described earlier. We consider the above as further support for the view that our framework leads to a better understanding and, more importantly, prediction of the feeding behaviour of animals.

\section{Discussion and conclusions}

There are many instances where one would be interested in understanding and predicting the feeding behaviour of animals. In farm animals, for example, one is interested in predicting the feeding behaviour which would sustain a certain level of production. In more extensively kept animals, one is interested in predicting the impact of the foraging animal on its environment through its feeding behaviour. Provenza and his coworkers do not seem to share the same desire for accurate prediction of feeding behaviour. In a recent investigation into the conditioned responses of similar sheep towards flavoured foods associated with the same levels of nutrient dose it was stated: "Lambs preferred the flavours paired with urea after the first and third conditioning periods. We cannot explain why preferences were not evident ... in the second period" (Villalba \& Provenza, 1997). In a similar investigation they state: "Doses of sodium propionate that condition preferences in some lambs condition aversions in some others" (Villalba \& Provenza, 1996; Provenza et al. 1998). In either case, no framework is invoked $a$ priori to predict such different feeding responses; the framework of the selforganization of behaviour is invoked for such differences a posteriori.

It is perhaps this difference in studying feeding behaviour that leads to the different approaches. We have stipulated that in order to be able to predict the feeding behaviour of animals we need to understand what they are trying to achieve through it. Current debate on the study of feeding behaviour lies in the nature of such goals (Tolkamp \& Ketelaars, 1992; Emmans \& Kyriazakis, 1995; Illius \& Jessop, 1996). Interestingly, in his only quantitative approach to feeding behaviour in the literature, Provenza also feels the need to invoke a teleonomic goal (that of fitness) in order to be able to make predictions (Provenza \& Cincotta, 1993). For the purposes of our framework, one needs to describe the maximum output rates which animals are seeking to achieve when they are given access to one or more foods where the composition of them or of their mixture is nonconstraining. The capacities of animals to cope with constraints will similarly need to be defined when they are given access to one or more constraining foods. Both the maximum output rates and the ability to cope with constraints will depend on the existing or current state of the animal, which will also have to be defined in terms which are consistent with the theory (Emmans \& Kyriazakis, 1995; Kyriazakis \& Emmans, 1998). Because we present our theory in the above terms, its predictions are made explicit and could be compared with outcomes from the real world. We view our framework of feeding behaviour as both evolving and dynamic in its nature, so we expect that it would eventually be modified or even replaced by a better one; we accept that this is the nature of progress in science. 


\section{References}

Arsenos, G. \& Kyriazakis, I. (1998). The continuum between preferences and aversions for flavoured foods in sheep conditioned with administration of casein doses. Animal Science (in press).

Bazely, D. R. (1990). Rules and cues used by sheep foraging in monocultures. In Behavioural Mechanisms of Food Selection, pp. 343-367 [R. N. Hughes, editor]. London: Springer-Verlag.

Cooper, J. J., Emmans, G. C. \& Friggens, N. C. (1994). Effect of diet on behaviour of individually penned lambs. Animal Production 58, 441 (abstr.)

Dardaillon, M. (1989). Age-class influences on feeding choices of free-ranging wild boars (Sus scrofa). Canadian Journal of Zoology 67, 2792-2796.

Day, J. E. L., Kyriazakis, I. \& Lawrence, A. B. (1986). An investigation into the causation of chewing behaviour in growing pigs: the role of exploration and feeding motivation. Applied Animal Behaviour Science 48, 47-59.

Day, J. E. L., Kyriazakis, I. \& Rogers, P. J. (1998). Food choice and intake: towards a unifying framework of learning and feeding motivation. Nutrition Research Reviews 11, $25-43$.

de Jong, A. (1981). Short- and long-term effects of eating on blood composition in free-feeding goats. Journal of Agricultural Science 96, 659-668.

Emmans, G. C. \& Kyriazakis, I. (1995). The idea of optimisation in animals: uses and dangers. Livestock Production Science 44, 189-197.

Friggens, N. C., Nielsen, B. L., Kyriazakis, I., Tolkamp, B. J. \& Emmans, G. C. (1998). Effects of food composition and stage of lactation on the short-term feeding behavior of dairy cows. Journal of Dairy Science (in press).

Hull, D. (1974). Philosophy of Biological Science. Englewood Cliffs, NJ: Prentice-Hall.

Illius, A. W. \& Jessop, N. S. (1996). Metabolic constraints on voluntary intake in ruminants. Joumal of Animal Science 74, 3052-3062.

Inglis, I. R., Forkman, B. \& Lazarus, J. (1997). Free food or earned food? A review and fuzzy model of contrafreeloading. Animal Behaviour 53, 1171-1191.

Kyriazakis, I. (1997). The nutritional choices of farm animals: to eat or what to eat? In Animal Choices, pp. 55-65 [J M Forbes, T. L. J. Lawrence, R. G. Rodway and M. A. Varley, editors]. Edinburgh: British Society of Animal Science.

Kyriazakis, I. \& Emmans, G. C. (1998). Voluntary food intake and diet selection. In A Quantitative Biology of the Pig [I. Kyriazakis, editor]. Wallingford: CAB International (in press).

Kyriazakis, I., Emmans, G. C. \& Whittemore, C. T. (1990). Diet selection in pigs: choices made by growing pigs given foods of different protein concentrations. Animal Production 51, 189-199.

Kyriazakis, I. \& Oldham, J. D. (1993). Diet selection in sheep: the ability of growing lambs to select a diet that meets their crude protein (nitrogen $\times$ 6.25) requirements. British Journal of Nutrition 69, 617-629.

Mach, E. (1960). The Science of Mechanics: a critical and historical account of its development (original English translation published 1893). LaSalle: Open Court Publishing.

Mayr, E. M. (1964). The evolution of living systems. Proceedings of the National Academy of Sciences of the USA 51 , 934-941.

Mayr, E. M. (1988). Towards a New Philosophy of Biology: observations of an evolutionist. Cambridge, MA: Belknap Press of Harvard University Press.

Nielsen, B. L., Lawrence, A. B. \& Whittemore, C. T. (1995). Effects of group-size on feeding behaviour, social behaviour, and performance of growing pigs using single-space feeders. Livestock Production Science 44, $73-85$.

Ørskov, E. R. (1988). Protein Nutrition in Ruminants, 2nd edn. London: Academic Press.

Provenza, F. D. (1995). Postingestive feedback as an elementary determinant of food preference and intake in ruminants. Journal of Range Management 48, 2-17.

Provenza, F. D. \& Cincotta, R. P. (1993). Foraging as a self-organization learning process: accepting adaptability at the expense of predictability. In Diet Selection, pp. 78-101 [R N Hughes, editor]. London: Blackwell Scientific Publications.

Provenza, F. D., Villalba, J. J., Cheney, C. D. \& Werner, S. J. (1998). Self-organization of foraging behaviour: From simplicity to complexity without goals. Nutrition Research Reviews 11, 199-222.

Sibly, R. M. (1975). How incentive and deficit determine feeding tendency. Animal Behaviour 23, 437-446.

Sibly, R. M. \& McCleery, R. H. (1976). The dominance boundary method of determining motivational state. Animal Behaviour 24, 108-124.

Tolkamp, B. J., Day, J. E. L. \& Kyriazakis, I (1998). Measuring nutrient intake in farm and laboratory animals. Proceedings of the Nutrition Society 57, 313-319.

Tolkamp, B. J. \& Ketelaars, J. J. M. H. (1992). Toward a new theory of feed intake regulation in ruminants. 2. Costs and benefits of feed consumption: an optimisation approach. Livestock Production Science 30, $297-317$.

Tolkamp, B. J. \& Kyriazakis, I. (1997). Measuring diet selection in dairy cows: effects of training on choice of dietary protein level. Animal Science 64, 197-207.

Villalba, J. J. \& Provenza, F. D. (1996). Preference for flavoured wheat straw by lambs conditioned with intraruminal administrations of sodium propionate. Joumal of Animal Science 74, 2362-2368.

Villalba, J. J. \& Provenza, F. D. (1997). Preference for flavoured foods by lambs conditioned with intraruminal administrations of nitrogen. British Journal of Nutrition 78, 545-561. 